\title{
Mejoramiento del proceso de enseñanza-aprendizaje de la matemática en la educación inicial: un desafío para la gestión de la educación
}

\section{Teaching and learning improvement of mathematics in early childhood education, a challenge for management education}

\author{
Recibido 07 marzo 2012 • Aceptado 25 junio 2012 • Corregido 28 junio 2012
}

\author{
María Marta Camacho Álvarez ${ }^{1}$ \\ Directora de la Escuela de Formación Docente, \\ Universidad de Costa Rica \\ camachomaria_marta@hotmail.com
}

\begin{abstract}
Resumen. Este artículo es producto de la investigación denominada: Mejoramiento del proceso de enseñanza-aprendizaje de la matemática en la educación inicial: vista desde la perspectiva docente, cuyo objetivo general fue analizar la formación del docente de preescolar en ejercicio, en lo relativo a los procesos de enseñanza y aprendizaje de la matemática, fortalezas, debilidades y necesidades para generar una propuesta metodológica que pueda fortalecer los procesos de formación docente y autoformación. Se presenta a su vez una reflexión desde la administración de la educación, como un desafío que desde esta disciplina debe asumirse en el mejoramiento de la enseñanza-aprendizaje de la matemática en la educación inicial. Destaca aspectos relacionados con la formación, la metodología aplicada en los cursos para formar a los educadores, los mecanismos de información utilizados de manera más frecuente, así como aspectos de los procesos de capacitación, desarrollo profesional y autoformación en los que participa el personal académico de educación inicial. Se describen brevemente algunas acciones tendientes al cambio en la cultura escolar en torno al proceso educativo en el ámbito de la matemática; siendo precisamente la cultura escolar un campo donde la administración de la educación asume un rol fundamental. Se hace un llamado de atención sobre la necesidad de que las instituciones universitarias brinden una formación más abarcadora, y que el resto de las instituciones dedicadas a procesos de desarrollo profesional de los educadores de las matemáticas mejoren los procesos de divulgación y capacitación, así como que la administración de la educación asuma estos procesos dentro de sus políticas prioritarias.
\end{abstract}

Palabras claves. Educación preescolar, educación matemática, estrategias didácticas, matemáticas, gestión de la educación y administración educativa

1 Doctora en Educación con énfasis en Administración Educativa de la Universidad Latina de Costa Rica. Posee una Licenciatura en Educación Preescolar de la Universidad de Costa Rica (UCR) y un Bachillerato en Ciencias de la Educación con énfasis en Educación Preescolar en la Universidad de Costa Rica (UCR). Actualmente es la Directora de la Escuela de Formación Docente de la Facultad de Educación de la Universidad de Costa Rica. 
Abstract. This article results from research entitled: Improvement of the teaching and learning of mathematics in early childhood education: A view from the teaching, whose overall objective was to analyze preschool teacher training, related to teaching and learning mathematics processes, strengths, weaknesses and needs to generate a methodology that can strengthen through teacher training, including self-training. This reflection since management is presented as a challenge that this discipline must be assumed to improve mathematics teaching - learning processes in early childhood education. Leading aspects training such as: courses methodology to train teachers, mechanisms used more frequently aspects of training processes; as well as, professional development and self- training in academic staff of early childhood education. Some actions aimed at changing school culture are briefly describe around learning process in mathematics, where school culture; specifically, educational management assumes a fundamental role. To conclude, the need for academic institutions provide more comprehensive training, and other institutions involved in professional development for mathematics teachers to improve processes of disclosure and training; aside from, educational management takes these processes into policy priorities.

Keywords. Preschool education, mathematics education, teaching strategies, math, educational management and educational administration.

\section{Presentación}

La administración de la educación asume un papel estratégico y relevante en el desarrollo de los procesos de enseñanza-aprendizaje de las organizaciones educativas; no es posible concebir la desarticulación de estos procesos con los objetivos institucionales desde la gestión de la educación.

De ahí, que la administración de la educación está llamada a ofrecer las condiciones institucionales y liderar los cambios organizacionales necesarios para que las diferentes propuestas educativas se desarrollen en forma óptima y, de esta forma, aportar al mejoramiento de la educación en general.

El área de matemática es una de las grandes debilidades del sistema educativo costarricense, por lo que le corresponde a la administración de la educación comprender a cabalidad su desempeño como un todo y establecer planteamientos que apoyen los procesos educativos y promuevan su aprendizaje; así, de esta forma ofrecer mejores resultados educativos a la sociedad.

La investigación en la que se sustenta este artículo es denominada Mejoramiento del proceso de enseñanza-aprendizaje de la matemática en la educación: vista desde la perspectiva docente, realizada en el Instituto de Investigaciones en Educación de la Universidad de Costa Rica, cuyo objetivo general fue analizar la formación del docente de preescolar en 
ejercicio, en lo relativo a los procesos de enseñanza y aprendizaje de la matemática, a partir de la identificación de fortalezas, debilidades y necesidades para generar una propuesta metodológica que fortalezca los procesos de formación docente y autoformación. Por tanto, este artículo pretende dar algunos aportes en este ámbito desde el papel de la administración de la educación.

\section{Antecedentes y sustento teórico}

Los antecedentes de los resultados obtenidos en los últimos años de intervención educativa en el área de la matemática, determinan como necesidades básicas por investigar: la revisión de contenidos programáticos, la necesidad de material didáctico, asesoramientos para mejorar la enseñanza, eficacia de los procedimientos de evaluación y promoción, actitud del educando y desarrollo de un currículo de matemática de acuerdo con los requerimientos nacionales y regionales.

Se han realizado varios estudios para analizar los efectos de la actitud y participación docente en los niveles de primaria y de secundaria, en relación con el área de la matemática; en ellos se ha examinado el papel del administrador de la educación, del educando, del educador, su formación y hasta el papel de los padres. Al respecto, se considera necesario y oportuno realizar evaluaciones en el nivel de la educación Inicial. Hay tres puntos centrales que se pueden retomar desde la gestión de la educación:

- Reconocimiento de los efectos que produce la educación temprana en el niño, en especial en los primeros seis años de su formación: tomando en cuenta que en la edad preescolar se forman muchas de las actitudes, predisposiciones y conocimientos básicos para la construcción de aprendizajes significativos y la importancia de la participación de los docentes y de los padres en este proceso. Tal y como lo señalan Gamboa (1994) y Hurlock (1999), asume relevancia una acertada educación en los primeros años de vida del infante, así como organizaciones educativas que conduzcan estos procesos con eficientes estrategias de gestión. Los responsables de liderar el proceso educativo en las organizaciones educativas muestran la excelencia en su rol de gestores al promover y desarrollar eficientes espacios educativos para que el proceso enseñanza-aprendizaje, desde los niveles primarios como lo es la educación preescolar, asuma protagonismo en estos procesos de formación, de manera que 
Revista Cientifica Digital ISSN:2215-2288

[Número publicado el 30 de J unio del 2012]
URL:http :/ / revista dig ita l.ea e.fc s.uc r.ac .cr/

el sistema educativo concrete y operacionalice su propuesta educativa como lo exponen Garbanzo y Orozco (2010), al referirse a este tema desde el papel del sistema educativo mediante la gestión:

El sistema educativo concreta su proyecto educativo mediante las organizaciones educativas distribuidas en diferentes niveles, a saber: preescolar, primaria, secundaria y educación universitaria; eso sí, se requiere de un acertado liderazgo que promueva las condiciones de autodesarrollo profesional en forma competitiva como única respuesta a las demandas de por sí exigentes del contexto en el que a la educación hoy le ha correspondido actuar; relaciones laborales/organizacionales de por sí complejas. (p.18)

Los períodos prenatal, infancia y primera infancia (antes del nacimiento hasta los seis años aproximadamente) revisten especial importancia porque es en ellos donde se cimientan las bases del desarrollo futuro del individuo (Hurlock, 1999). Cada persona trae un bagaje genético definido, sigue un proceso maduracional bastante predecible pero al mismo tiempo individual, de manera que cada niño se desarrolla a su propio ritmo. Es decir, el proceso de desarrollo se da dentro de los límites establecidos por la genética, siendo que el resultado final depende también del ambiente. Así, todo proceso educativo debe partir de las características del educando y su medio físico, natural y social, lo que considera que los procesos educativos se acompañen, para su adecuada implementación, de un liderazgo institucional de sus gestores con alto nivel de responsabilidad social, en lo que le corresponde dentro de este contexto de centro educativo. Para Venegas Jiménez (2005), estos líderes deben tener una gran responsabilidad en la formación de las personas:

Los líderes educativos tienen por compromiso fortalecer y mejorar el sistema educativo. Como costarricenses, tienen la responsabilidad de forjar una niñez y juventud ávidos de conocimientos, de ideas novedosas, pero convencidos de un protagonismo en la construcción de un mundo más justo y tolerante. Por esto es que se hace evidente la necesidad de contar con líderes en la Educación que enfaticen las exigencias de una educación de calidad, eficaz y eficiente, según las características, necesidades e intereses de su comunidad, en su contexto social, económico y político. Se necesita de 


\section{Givestión \\ Escuela de Administración Educativa}

[Número publicado el 30 de J unio del 2012]
Revista Cientifica Digital ISSN:2215-2288

URL:http:// revista digita l.eae.fcs.ucr.ac.cr/

líderes educativos que respiren plenamente la realidad social, que fomenten el trabajo en equipo, bajo la consigna de servir antes de ser servido. (pp. 9-10)

De esta forma, se evidencia la importancia de que los sistemas educativos cuenten con acertados liderazgos que sepan conducir, con alto nivel de responsabilidad, las distintas propuestas educativas, encaminadas a mejorar la calidad de la educación que se imparte.

- Formación docente: La Coordinación Educativa y Cultural Centroamericana se ha especializado en la investigación relacionada con la formación de docentes en esta región de América. De ahí, que se destaque el Informe de Centroamérica en el Foro Mundial de Educación para Todos (UNESCO, 2000); los Perfiles y planes de estudio del docente de la Educación Primaria o Básica de los países de Centroamérica: estudio comparativo de Venegas (2001); el Proyecto de establecimiento de estándares para la educación primaria en Centroamérica, realizado en 1999; así como el referente a Logros y desafíos de Centroamérica de Santiago a Quebec II, reunión de Ministros de Educación de las Américas (CIDI, 2001).

Específicamente para Costa Rica, se tiene como antecedentes la investigación denominada Formación Inicial de docentes de educación primaria o básica. Estudio Nacional de Costa Rica, realizada por Venegas en el año 1996; la propuesta de Costa Rica Perfil de formador de docentes de educación primaria o básica. Proyecto apoyo al mejoramiento de la formación inicial de docentes de la educación primaria o básica, Orozco (2000). Las estrategias didácticas empleadas en la enseñanza del área pedagógica, en los planes de formación inicial de docentes para la educación primaria, en las universidades estatales costarricenses, realizada por Hernández, Montenegro, Francis y Gonzaga, en el año 2002, también se encuentra entre estas iniciativas.

Se han preparado diversos estudios sobre la formación de los educadores y los asesoramientos que reciben. Sin embargo, están enfocados al nivel de la educación básica y diversificada y no hacia la preescolar, tal es el caso de las investigaciones antes mencionadas y las cuatro realizadas por Esquivel, Delgado y Peralta (1983).

Siendo el docente de preescolar el primer agente que promueve experiencias de educación formal en relación con las matemáticas, es de suma importancia identificar las áreas débiles y fuertes de su formación académica, aspecto que afectará la planificación, desarrollo y 
evaluación del proceso educativo. En este campo, en el nivel institucional, es posible liderar procesos de diagnóstico académico desde la administración de la educación y, de esta forma, promover procesos de capacitación profesional.

Con respecto a la formación y asesoramiento docente, investigaciones realizadas refieren las principales dificultades que tienen los docentes para enseñar matemáticas en los jardines de infantes; en las que las docentes consideran útil la formación recibida durante sus años de estudio; sin embargo, la mayoría de los sujetos nunca han recibido capacitación para la enseñanza de esta ciencia.

Al respecto, la investigación realizada por Esquivel, Delgado y Peralta, en (1983), denominada Diagnóstico evaluativo de la enseñanza de la matemática en la educación general básica y educación diversificada, señala que las docentes tienen pocas oportunidades de recibir asesoramiento y la labor de los asesores no se lleva a cabo satisfactoriamente, además, de que el planeamiento que realizan los docentes es deficiente. En la investigación de Esquivel, Delgado y Peralta (1983), denominada ¿Cuál es la formación académica y situación laboral de los maestros y profesores de matemáticas de la educación general básica y la educación diversificada?, se determinó que los docentes llegan hasta el nivel de profesorado y bachillerato y son pocos los que continúan procesos de especialización, tanto en el área de la matemática como en ciencias de la educación.

Esquivel, Delgado y Peralta (1983) realizaron otro estudio sobre los asesoramientos que los docentes reciben, en el que se determinó que el personal que asesora en la enseñanza de la matemática, en la Educación General Básica y Educación Diversificada, cuenta con pocos años de experiencia en la labor de asesoramiento; en su mayoría, se ha especializado más en el área de la administración educativa que en la de matemática.

Los resultados de los estudios nacionales relacionados con los logros que alcanzan los alumnos en la materia de matemáticas y las evaluaciones realizadas a los procesos de enseñanza-aprendizaje de la matemática en Educación Básica y Diversificada, así como los resultados de las pruebas de bachillerato, no pueden reflejar la situación del proceso de enseñanza-aprendizaje en preescolar. Cabe resaltar que muchas veces las deficiencias se buscan en los niveles intermedios y superiores, cuando las primeras raíces del problema pueden estar en las bases. Estos planteamientos coinciden con lo expuesto por Gil y Guzmán (1991): 
La adecuación curricular, en el ámbito de la matemática, no es pues una simple adaptación del currículo existente a grupos de personas, situaciones determinadas o contextos socio-culturales particulares por razones de progreso pedagógico y científico, se trata de un cambio profundo en todo lo que es, implica, genera y conlleva el currículo matemático. (p.12)

Son retos para las personas e instituciones involucradas en la administración de la educación, en el área de la educación preescolar y específicamente en el área de la matemática, el fortalecimiento de la fundamentación pedagógica y metodología, el impacto en políticas nacionales de desarrollo educativo, la construcción, de manera conjunta, por un lado del perfil del educador o educadora formador de docentes y por otro lado, el perfil del profesional en educación preescolar. También, el mejoramiento del liderazgo de la condición profesional y laboral de los educadores, mediante el desarrollo de programas que estimulen e incentiven el desempeño docente, la implementación de procesos de desarrollo profesional, nuevas estrategias didácticas que contribuyan a la calidad de los aprendizajes, la creación de instituciones educativas para la población de niñas y niños menores de cuatro años que permitan el acercamiento con la matemática desde edades tempranas, la construcción de aprendizajes significativos en las asignaturas del currículo de matemática, la promoción de innovaciones educativas, la realización de investigaciones en el aula que sustente la toma de decisiones para el desarrollo y fortalecimiento del sistema educativo, así como la concreción de nuevas formas de participación en el sistema educativo de la familia y las instituciones. Para el logro de todo lo anterior, se debe involucrar a todos los actores que intervienen en la gestión de la educación.

Por su parte, la formación del docente en educación inicial en el área de la matemática, se concibe como el proceso de formación y capacitación del docente en el área de matemática. Para Álvarez, Fernández y Ruiz (2011):

La formación universitaria del docente consiste en el seguimiento de un periodo en la enseñanza estricta y rigurosa, bajo la educación de profesores especializados en diferentes áreas que integran la formación docente, durante un lapso o período determinado, en una institución educativa de enseñanza superior. Esto con el fin de adquirir las bases teóricas pedagógicas que se requieren en la docencia. 
Este período de seguimiento se articula a procesos de calidad de la educación que se ofrece, donde el papel docente es relevante, en el cual intervienen múltiples factores, tal y como lo expone Gonzaga (2005):

La calidad de la Educación Primaria depende de múltiples factores, uno de los más importantes es la formación de docentes. Por lo que se requiere contar con un docente creativo, que posea un conocimiento amplio y profundo de lo qué, cómo y cuándo debe enseñar; con un manejo apropiado de estrategias de enseñanza y aprendizaje, de procedimientos e instrumentos de evaluación, y con una clara comprensión de lo que significa su trabajo de aula, tanto en el ámbito del desarrollo individual y grupal de los estudiantes, como del impacto social de la labor educativa, capaz de vencer limitaciones y obstáculos y de llevar a cabo una práctica docente que satisfaga las expectativas del sistema educativo y de la sociedad en general. (p.2)

Dentro de la calidad de la educación, asume relevancia el conocimiento del personal docente que tiene a cargo el proceso enseñanza-aprendizaje; manifestándose el conocimiento de las docentes en relación con los temas propios del área de la matemática en la educación inicial como el dominio que posee el docente a nivel teórico y práctico, en el campo de la matemática aplicados a la educación inicial. Para Tobón y Núñez (2006) el conocimiento es un conjunto de representaciones entretejidas basadas en información, con análisis, síntesis, interpretación y argumentación, en un determinado contexto, con significación y consecuencia de sus interrelaciones; además agregan que:

El conocimiento está en continua evolución y cambio por el aumento continuo de la información, acorde con el desarrollo de las diversas disciplinas y ciencias, y la evolución de las tecnologías de la información y la comunicación. (p.30)

Todo conocimiento es consecuencia de una secuencia de creaciones que se han ido produciendo y expandiendo a lo largo de los siglos, tal y como lo afirma Darceles (2007). Por su parte, la metodología utilizada por los docentes en el proceso de enseñanza aprendizaje de la matemática en la educación inicial refiere al conjunto de métodos, técnicas y actividades que 


\section{Gi:Gestión \\ Escuela de Administración Educativa}

[Número publicado el 30 de J unio del 2012]
Revista Cientifica Digital ISSN:2215-2288

URL:http :// revista digita l.eae.fcs.ucr.ac.cr/

utilizan los docentes en el proceso de enseñanza-aprendizaje de la matemática en la educación inicial.

En el escenario educativo el docente es aquella persona que tiene como función enseñar, es el mediador y mediadora formados para guiar y acompañar a la población estudiantil en la adquisición de los conocimientos que se requieren para la vida; su función de educar consiste en encaminar, acompañar y desarrollar las facultades morales, intelectuales y físicas del estudiante, con el fin de posibilitar su desarrollo como sujeto. Al respecto, Zambrano (2002, p.114.) expone que "educar será ante todo, guiar al que empieza a vivir en esta su marcha responsable a través del tiempo", de ahí la importancia de la metodología que se siga.

En este contexto, asume relevancia la formación del docente de educación inicial en el área de la matemática, la cual para la investigación supracitada se entiende como el proceso de formación y capacitación que realiza el o la docente de educación inicial, en el área de la matemática. Incluye recursos, métodos y estrategias de evaluación más utilizadas, así como fortalezas, debilidades y necesidades (Martín, 1999).

En cuanto a los conocimientos de las y los docentes en relación con los temas propios del área de matemática en la educación inicial, estos se conciben como aquel dominio que tienen en el nivel teórico y práctico, en relación con los diversos temas propios de área de la matemática en la educación inicial.

Finalmente, la metodología utilizada por los y las docentes en el proceso de enseñanzaaprendizaje de la matemática en la educación inicial, se define como el conjunto de métodos, técnicas, actividades que utilizan los docentes en el proceso de enseñanza-aprendizaje de la matemática en la educación inicial.

\section{Metodología}

La investigación se realizó con un enfoque cualitativo, con un método compuesto por seis momentos que se organizaron en seis fases: la preparatoria, acercamiento teórico al

problema, acercamiento práctico, acercamiento teórico-práctico al problema, informe de investigación y propuesta.

Estas fases estaban compuestas por diversas etapas que, a su vez, se subdividieron en actividades, para finalizar en la definición de los productos esperados para cada momento y fase. 
Revista Cientifica Digital ISSN:2215-2288

URL:http :// revista digita l.eae.fcs.ucr.ac.cr/

[Número public ado el 30 de J unio del 2012]

Los objetivos específicos que se plantearon estaban dirigidos a construir un acercamiento teórico-práctico al campo de la formación del docente de educación preescolar en el área de la matemática, para el conocimiento de su estado actual y el establecimiento de algunos roles, perfiles, actitudes. A determinar con los y las docentes los procesos de formación en el área de matemática para generar una propuesta metodológica que pueda fortalecer los procesos de formación docente y autoformación. A la información recogida en el campo y en la literatura en relación con la formación del docente de preescolar para generar una propuesta metodológica que pueda fortalecer los procesos de formación docente y autoformación. A socializar con los y las docentes de preescolar la información recolectada y procesada, tanto desde un enfoque teórico como práctico, para hacer conciencia de la realidad, actuar sobre ella y transformarla y finalmente, a generar una propuesta para un proyecto de Acción Social (Extensión Docente), que retome procesos de autoformación docente en el área de la matemática en preescolar.

Con el propósito de lograr los objetivos propuestos, se recurrió a fuentes primarias y secundarias, también se visitaron instancias nacionales e internacionales que abordan el tema de la educación matemática. Las fuentes primarias la constituyen 36 miembros del personal docente y administrativo de doce jardines infantiles de la provincia de Heredia.

El personal docente y administrativo participante de la investigación, son mujeres, de las cuales el 78,32\% tienen edades entre los 20 y los 40 años. Un $56,11 \%$ de la población tiene cinco o más años de experiencia laboral, lo que es un aspecto importante ya que indica que existe una experiencia previa en docencia en la educación Inicial; además, sus visiones incluyen años de participación en procesos formales e informales de capacitación. De los participantes en el estudio un $66,66 \%$ ha concluido estudios de bachillerato o licenciatura, siendo solo un $20 \%$ los que tienen estudios de diplomado y un $13,88 \%$ posee una maestría. Esta información recalca que las docentes han cumplido con procesos formales de educación superior.

Las fuentes secundarias o de segunda mano estaban constituidas por bibliografía, documentos de otras investigaciones, artículos, información obtenida por medio de la red de Internet, entre otros.

Las categorías de análisis que se determinaron en la investigación fueron la formación de la docente de educación inicial en el área de la matemática, el conocimiento de las docentes en relación con los temas propios del área de matemática en la educación inicial y la 


\section{Revista Cientifica Digital ISSN:2215-2288}

[Número publicado el 30 de J unio del 2012]
URL:http :/ / revista dig ita l.ea e.fc s.uc r.ac .cr/

metodología utilizada por los docentes en el proceso de enseñanza-aprendizaje de la matemática en la educación inicial; componentes que permitieron en este estudio una reflexión de los desafíos al respecto que presenta la administración de la educación. Estas categorías se sintetizan seguidamente:

- Formación del docente en educación inicial en el área de la matemática: proceso de formación y capacitación del docente en el área de matemática, bajo enfoques especializados e innovadores y un manejo apropiado de estrategias de enseñanza y aprendizaje (Álvarez, Fernández y Ruiz, 2011).

- Conocimiento de las docentes en relación con los temas propios del área de la matemática en la educación inicial: se considera como el dominio que posee el docente en el nivel teórico y práctico, en el campo de la matemática, aplicados a la educación inicial. Conjunto de representaciones articuladas sustentadas en información, con análisis, síntesis, interpretación y argumentación, en un determinado contexto (Tobón y Núñez, 2006).

- Metodología utilizada por los docentes en el proceso de enseñanza aprendizaje de la matemática en la educación inicial: Conjunto de métodos, técnicas, actividades que utilizan los docentes en el proceso de enseñanza-aprendizaje de la matemática en la educación inicial. Refiere a las acciones en función de la enseñanza que guía y acompaña a la población estudiantil en la adquisición de los conocimientos (Zambrano, 2002).

Para la realización de esta investigación, se emplearon básicamente cuatro instrumentos que responden a métodos de interrogación, cuyos resultados luego fueron analizados en pequeños grupos; posteriormente, en el gran grupo y finalmente, por la investigadora. Antes de las sesiones de aplicación de instrumentos se realizaron sesiones de validación con apoyo de especialistas, según criterio de expertos.

\section{Desarrollo y análisis de resultados}

La mayoría de investigaciones que se han realizado en el área de la matemática están enfocadas al nivel de educación básica y diversificada y no hacia la educación preescolar, por lo 


\section{Revista Cientifica Digital ISSN:2215-2288}

[Número publicado el 30 de J unio del 2012]

URL:http:// revista digita l.eae.fcs.ucr.ac.cr/

que se considera indispensable abarcar por lo menos dos de las prioridades de investigación en la enseñanza de la matemática: la formación y los procesos de autoformación, así como aspectos metodológicos, enfocados hacia el nivel de educación inicial o preescolar.

Con respecto de la formación universitaria que reciben, al entrevistar a la población docente participante en el presente estudio en lo relativo a las lecciones de matemáticas que recibió en la universidad, las entrevistadas indican que el plan de estudios que ellos llevaron contenía solo un único curso de matemática para preescolar y que este a su vez era o muy práctico o muy teórico, lo que sugiere dos aspectos relevantes: la necesidad de establecer un balance entre los aspectos teóricos y prácticos que brinda el curso de matemática para preescolar, y saca a relucir que el plan de estudios de Educación Preescolar en las universidades públicas y privadas contempla solo un curso de matemáticas. Por lo anterior, se recomienda el mayor aprovechamiento de los recursos humanos, bibliográficos, investigativos y de tiempo, con el fin de que el estudiante pueda disponer de más herramientas que le aseguren el éxito en el desempeño de su función docente.

En este sentido, un proceso importante, en lo relacionado con la formación universitaria, lo externa Leibowicz (2000) al indicar que:

La formación inicial se une a la formación continua en un espiral de procesos que propicia la gestación de una capacidad sostenible de aprendizaje. Se trata de dar lugar a una continuidad creciente de acciones de aprendizaje que garanticen no solo un empleo como resultado de la formación inicial, sino el despertar de una «pasión» por aprender que se mantenga a lo largo de la vida activa y contribuya a aumentar la competitividad en el trabajo. (p.8)

En cuanto a la metodología aplicada en los cursos que las docentes recibieron en la universidad, en torno a los procesos de enseñanza-aprendizaje de la matemática a nivel inicial, cabe destacar que las actividades y técnicas que más se ejecutaron durante el proceso de formación universitaria en el curso de matemáticas para la carrera de Educación Preescolar, (de acuerdo con lo que mencionan las personas involucradas en el estudio) son la elaboración de materiales, estudio de conceptos, teorías, lecturas, juegos, actividades, exposiciones, trabajos en grupo y la realización de pruebas cortas o exámenes. 


\section{Gi:Gestión \\ Escuela de Administración Educativa}

[Número publicado el 30 de J unio del 2012]
Revista Cientifica Digital ISSN:2215-2288

URL:http:// revista digita l.eae.fcs.ucr.ac.cr/

Para enriquecer los procesos de docencia universitaria en el caso de los cursos de matemáticas para la Educación Inicial, se sugiere complementar con otras técnicas tales como la formación de equipos de trabajo cooperativos, la resolución de problemas, situaciones o dudas, resúmenes orales o explicitaciones de conocimientos previos sobre un tema antes de iniciarlo, realización, análisis y evaluación de lecturas, grupo de discusión para determinar similitudes, diferencias, creencias, mitos, interrogantes relacionados con un tema, constructo, idea o experiencia, debate o discusiones guiadas con temas que los estudiantes ya conocen, investigan o se informan previamente. Además el estudio de casos, visitas a instituciones de educación, observaciones en el aula, representación de roles de situaciones de la vida real de acuerdo con unas características indicadas previamente, representación de un juicio o una discusión de ideas con dos grupos contrapuestos, esto relacionado con un tema específico; exponer resultados de investigaciones recientes, creación de recursos didácticos, compartir ideas en parejas o pequeños grupos, panel con especialistas, realización y participación en talleres y la Técnica del Grupo Nominal (TGN) en la que el profesor o los estudiantes proponen un tema o problema por resolver individualmente. Luego se pide a los participantes, por turno, que digan una de las ideas, se anotan en la pizarra, posteriormente se jerarquizan y se realiza un resumen, entre otras técnicas.

En lo relativo a la actitud de los docentes universitarios con los que recibieron el curso de matemática para la educación preescolar, los participantes del estudio en mención expresaron que algunos recibieron lecciones presenciales y otros a través de tutorías por el sistema de Educación a Distancia. Además, en las respuestas se pueden evidenciar dos vertientes claramente definidas: aquellos que fueron partícipes de un proceso conductista (magistral y de poca interacción) y otro grupo que participó en un proceso más constructivista. Al respecto, Santiago (2006), expresa en relación con el conductismo:

Lo descrito facilita apreciar la verdad entendida como la reproducción fiel de la imagen interior a la realidad que representa y su transmisión consistirá en promover su copia de la manera más exacta, precisa y estricta. Por esta razón el conocimiento que se obtiene debe ser neutral, imparcial y cierto. (p.150)

El enfoque conductista considera que el conocimiento se genera a partir de los estímulos externos que se reciben de la realidad dada, la cual existe independientemente del sujeto. El 


\section{G Gestión G: $E$ Educación

[Número public ado el 30 de J unio del 2012]
Revista Cientifica Digital ISSN:2215-2288

URL:http:// revista digita l.eae.fcs.ucr.ac.cr/

sujeto reproduce la realidad tal y como es, sin intervenir ni construir su conocimiento.

Por otra parte, está el constructivismo para el que Onnetto (2004, p.38), "considera que el cerebro no es un mero recipiente donde se depositan las informaciones, sino una entidad que construye la experiencia y el conocimiento, lo ordena y da forma". Por su parte, para Barreto, Gutiérrez, Pinilla y Parra (2006, p.15), "supone que el conocimiento es una construcción mental, resultado de la actividad cognitiva del sujeto que aprende, concibiendo el conocimiento como una construcción propia, que surge de las comprensiones logradas a partir de los fenómenos que se quiere conocer".

Con respecto de los mecanismos de información, las frecuencias de los datos (organizados con referencia a número de veces) demuestran que en su mayoría las docentes nunca reciben información de matemáticas enviada por instituciones gubernamentales o no gubernamentales en formato de circulares, boletines, Internet, asesoramientos, reuniones, talleres o conferencias y que la casilla muchas veces permanece vacía en todos los recursos propuestos. Al analizar este aspecto, se puede determinar la necesidad de generar este tipo de herramientas informativas, de crear espacios por medio de las técnicas propuestas u otras relacionadas con el avance de la ciencia, la tecnología y los sistemas de información modernos. Esto sin olvidar métodos tradicionales y masivos como son la prensa escrita, la radio y la televisión.

Ante estos resultados se debe llamar la atención de líderes ministeriales, asesores, supervisores, directores, coordinadores de áreas, especialistas en matemática, ya que se denota la poca expansión que se tiene en recursos de información que lleguen de manera continua, oportuna, constante, ágil y permanente a docentes. Asimismo, se denota la necesidad de que las instituciones gubernamentales o no gubernamentales, públicas o privadas dedicadas a procesos de formación, información y divulgación ofrezcan más servicios de distribución y promoción de material de calidad especializado en el área de la matemática. Importante en este campo también que la gestión de la educación asuma con alto nivel de responsabilidad social estas limitaciones y asumir acciones propositivas.

De acuerdo con los recursos de información relacionada con matemáticas que las docentes buscan por sus propios medios, se concluye que pocas veces buscan realizar reuniones de sección o de escuela, talleres y otros recursos como lo son el acceso a libros, artículos, comparar experiencias, revistas y enciclopedias.

Con respecto de los procesos de capacitación, desarrollo profesional y autoformación de 
Revista Cientifica Digital ISSN:2215-2288

URL:http://revistadigita l.eae.fcs.ucr.ac.cr/

acuerdo con la investigación supracitada, la tercera parte de los docentes participantes en el estudio, no recibieron asesoramiento de matemática ni por iniciativa de la institución donde laboran, ni por el Ministerio de Educación Pública (MEP), ni por iniciativa propia. Esto quizás porque no es frecuente que se den capacitaciones en el área de matemáticas a nivel de educación inicial y también por la gran limitante que hay de permisos en el tiempo laboral para asistir a procesos de desarrollo profesional. Menos de la mitad de las docentes indicaron que asisten a asesoramientos fuera del tiempo lectivo y aproximadamente una décima parte lo hacen tanto dentro como fuera del tiempo de trabajo en el aula. Sin embargo, es importante resaltar que la mayoría de personas sí recibieron asesoramiento.

Los factores que dificultan la participación en proceso de capacitación de acuerdo con los docentes se pueden agrupar en dos áreas: aquellos que tienen sus causas en factores intrínsecos del individuo y aquellos factores externos, ajenos o extrínsecos a él. Entre los factores intrínsecos o que tienen que ver con sus decisiones o sus capacidades, las docentes de este estudio mencionan básicamente dos: "se olvidan conceptos y procesos" y "falta de motivación". Los factores intrínsecos de una u otra forma se ven influidos por los extrínsecos. Por ejemplo, la calidad, variedad, cantidad de procesos de capacitación que se ofrecen, los estímulos que estos proponen a la docente tienen que ver también con la motivación que este tenga para asistir a ellos, para participar y para asimilar la información que se proporciona.

Al respecto, Longworth y Keith (1996) citados por Leibowicz (2000), consideran que la educación continua consiste en:

(... ) el desarrollo del potencial humano a través de un proceso de apoyo continuo que estimula y pone en manos de los individuos la capacidad para adquirir los conocimientos, valores, habilidades y actitudes que requerirán a lo largo de sus vidas y que aplicarán con seguridad, creatividad y placer en todos los papeles, circunstancias y contextos. (p.44)

Para Leibowicz (2000, p.43), en un sentido amplio del concepto, "la formación continua integra procesos de aprendizaje que están vinculados al trabajo y a la vida personal. Por lo tanto, abarca acciones intencionales e informales, y ambos tipos de formación merecen reconocimiento, sobre todo cuando son certificados". Lo que evidencia la importancia de la formación continua en los procesos de aprendizaje en general en aras de la calidad de la educación ofrecida. 


\section{GeGestión \\ Escuela de Administración Educativa}

[Número public ado el 30 de Junio del 2012]
Revista Cientifica Digital ISSN:2215-2288

URL:http:// revista digita l.eae.fcs.ucr.ac.cr/

Entre los factores extrínsecos, las participantes de la investigación exponen que "se ofrecen para escuelas públicas y no para privadas", "casi no se imparten cursos", "falta de información", "disposición de tiempo", "factor económico", "en las regiones no se dan asesoramientos", "el MEP no da permiso", "el horario alterno de las escuelas", y "que se imparten en muy pocas ocasiones".

Cabe destacar la responsabilidad que tienen las docentes en el proceso de autoformación y de formación continua, ya que no todos los recursos de información y capacitación van a llegar a sus manos, sino que él o ella tienen la responsabilidad de buscarlos, obtenerlos, adquirirlos, además de analizarlos y utilizarlos. No menos relevante el papel de la administración de la educación facilitando y desarrollando estos procesos de formación.

Sobre el tema del papel del profesional en el desempeño, Martin (1999) indica:

El concepto del término de profesión tal como lo empleamos hoy es un término descriptivo, el mismo conlleva una connotación ideológica. El profesional debe tener o cumplir el desempeño de una función social, para esto es crucial tener un grado de destreza basado en el conocimiento, con valores explícitos en un código, para lo cual es necesario una larga formación superior (p. 198)

Al respecto, tomando en cuenta esta particularidad, los profesores pasan a ser una figura clave en el cambio, específicamente en el área de la matemática se requiere por lo tanto:

\section{Conocimiento de la actividad matemática, de la historia y epistemología de la disciplina.}

No solo basta con saber qué se desea enseñar o qué se desea y necesita aprender. Lo ideal es conocer todos los fundamentos que intervienen en la historia, la evolución y desarrollo de un área, en este caso la matemática. Cada disciplina tiene su razón de ser y cada tema tiene su origen, por lo tanto es importante conocerlo, no para generar procesos de memorización de estos, sino para lograr una ubicación en un tiempo y en un espacio determinado, para comprender por qué surgen las disciplinas y cuál es su aplicación en la vida cotidiana. 


\section{Revista Cientifica Digital ISSN:2215-2288}

[Número publicado el 30 de Junio del 2012]

\section{Cambio en la cultura matemática escolar.}

Se requiere un cambio social en la manera de ver la matemática, ya que existen mitos y creencias relacionados con esta disciplina. La mayoría de ellos negativos, los cuales son reforzados de manera desequilibrada por los medios de comunicación social, la familia y hasta las instituciones educativas.

\section{Utilización de recursos de las ciencias de la información y la comunicación.}

En el mundo actual la información posee mucha relevancia y más que esta, recobra importancia el conocer y utilizar diferentes medios para accesarla. Esto implica que si bien es cierto, la información genera nuevos conocimientos y nuevas construcciones, es importante determinar cuál es relevante, cuál es necesaria, cuál es fiel, válida, científicamente comprobada y ampliamente analizada. Lo anterior, debido a que con el ingreso de las tecnologías de la comunicación son muchos los datos que circulan en el ambiente digital, virtual o físico, pero no todos ellos tienen un carácter formal, formativo, científico o investigativo.

El tener acceso a la información digital ha permitido que en diversas áreas geográficas se puedan generar indagaciones, investigaciones, procesos de divulgación e incluso procesos de transformación personal, profesional y educativa que antes estaban limitados para personas de áreas más céntricas que tenían acceso a bibliotecas.

Saber discriminar el tipo de información que se va a utilizar queda en manos de cada persona, de ahí que el papel de las instituciones públicas y privadas, servicios de biblioteca comunales y nacionales, universidades y centros de estudio debe tender a generar las herramientas para que tanto estudiantes como docentes aprendan a tener acceso a estos medios, a ser críticos y analíticos ante ellos.

\section{Conocimiento del currículo matemático escolar.}

Existen varias versiones de lo significa e implica el currículo algunas muy restringidas que lo enmarcan dentro del ámbito institucional o incluso en el aula. Otras más amplias que lo involucran con la comunidad local, regional, nacional y mundial, en el que participan todos los sujetos e instituciones. 


\section{Givestión \\ Escuela de Administración Educativa}

[Número publicado el 30 de Junio del 2012]
Revista Cientifica Digital ISSN:2215-2288

URL:http:// revista digita l.eae.fcs.ucr.ac.cr/

Desde una perspectiva restringida el currículo de matemática se limitaría al proceso de planificación y desarrollo de un programa de estudio definido, para un grupo delimitado de estudiantes y con un detalle específico de contenidos.

Partiendo de una perspectiva más amplia, el currículo de matemática involucra a todos los agentes y sujetos de la comunidad educativa y, más allá de ella, se relaciona con el mundo circundante, trasciende el tiempo y el espacio físico, tiene una razón dentro de la realidad existente y se involucra con la cotidianidad.

Relacionando este punto con el postulado anterior, se puede indicar que con una visión más amplia del currículo matemático se pueda dar significado a la razón por la cual la matemática surge como disciplina necesaria para el desarrollo de la vida humana, más que como una materia de un plan de estudio.

\section{Conocimiento de los estudiantes y de los procesos de aprendizaje.}

El proceso de enseñanza-aprendizaje de la matemática no puede verse como un elemento aislado del individuo o del grupo de individuos por el cual esta área del saber tiene razón de ser y para que cobre relevancia en la vida humana debe ser analizada, visualizada y entendida desde la perspectiva de la persona o grupo de personas al cual va dirigido el proceso. Por lo tanto, se hace necesario poseer un conocimiento de los estudiantes y de la manera como el individuo aprende según la edad, la madurez, las características físicas, sociales, afectivas, cognitivas y psicomotoras. Pensar en un proceso educativo sin tomar en cuenta al estudiante, es un error que trae como resultado procesos pocos significativos, no interesantes y poco fructíferos.

\section{Cambio en el papel de los conocimientos y creencias previas.}

Este postulado se refiere al aprovechamiento que debe hacer el docente y los estudiantes con respecto de los conocimientos, creencias y experiencias previas con que cuentan. El papel del docente que sabe y el alumno que aprende ha sido superado (quizás más en la teoría que en la práctica) por la concepción de que en el proceso educativo tanto el estudiante como el docente son activos contribuyentes. Lo anterior, entre otras cosas, es debido a todo el bagaje de ideas, pensamientos, vivencias, constructos que ambos poseen y que 
Revista Cientifica Digital ISSN:2215-2288

[Número public ado el 30 de J unio del 2012]

requieren compartir, presentar, reelaborar con el objetivo de que construyan nuevos aprendizajes y nuevas experiencias.

\section{Articulación de la teoría y la práctica.}

Los procesos de formación de los profesores de matemáticas deben articularse a través de la teoría y la práctica. No solo es suficiente tener un bagaje de conocimientos, saber acceder a ellos, organizarlos o analizarlos. Es necesario darles un sentido práctico, un uso específico, ubicarlos en un contexto real, de manera que sea atrayente y útil, que parta de una necesidad, de una realidad en la que el individuo está inmerso y en la cual él o ella puedan interactuar, comprender o aplicar.

\section{Utilización métodos activos que acerquen al estudiante con la realidad.}

Cada método tiene sus pros y sus contras y, en muchas ocasiones, no se puede delimitar el proceso educativo a uno solo de ellos. Sin embargo, el enfoque hacia métodos activos, participativos, en los que las y los estudiantes tienen un papel dinámico y protagónico, han generado como resultado aprendizajes más significativos.

El conocimiento matemático es una herramienta básica para la comprensión y manejo de la realidad en la que vivimos y por lo tanto es importante que el alumno manipule objetos matemáticos, active su capacidad intelectual, ejercite su creatividad, reflexione sobre su proceso de pensamiento, tenga confianza en sí mismo y se divierta. Otro aspecto fundamental es permitir que los estudiantes exploren, investiguen y tengan tiempo para que resuelvan lo que se les plantea.

\section{Creación de un ambiente físico y emocional agradable y acogedor.}

El ambiente es uno de los elementos fundamentales en el proceso educativo, el cual está compuesto no solo por el estado en que se encuentra el aula o el edificio, sino también los recursos, decoraciones, materiales, ubicación espacial, mobiliario con que se cuente, el color de las paredes, la ventilación, la iluminación natural y artificial, entre otros aspectos. 


\section{GeGestión \\ Escuela de Administración Educativa}

[Número public ado el 30 de J unio del 2012]
Revista Cientifica Digital ISSN:2215-2288

URL:http:// revista digita l.eae.fcs.ucr.ac.cr/

También influye el ambiente emotivo o afectivo, es decir, todos los sentimientos y emociones que se intercambian diariamente dentro y fuera de clase. Quizás este aspecto repercuta más en el aprendizaje que el ambiente físico, ya que traspasa las paredes de la escuela, las barreras del tiempo e influye en la vida personal y familiar. Se recomienda que la matemática se enseñe de una manera muy cercana a la realidad, propiciando un ambiente agradable y acogedor.

\section{Procesos de motivación hacia la matemática.}

Reflejar en sí mismo y generar en los estudiantes procesos de motivación hacia la matemática. "Nadie puede dar lo que no tiene" dice el refrán popular, y para poder motivar e interesar al alumno debe existir primero una gran fuerza motivacional interna. A pesar de que el docente no exprese verbalmente muchas de sus ideas, pensamientos, sentimientos, emociones, existe otro lenguaje más sutil y discreto que no se expresa con palabras sino más que todo con actos, expresiones posturales, faciales y actitudinales.

Ese lenguaje corporal debe ser congruente con el oral y escrito, debe estar en armonía, ya que los estudiantes sutilmente hacen lectura y confrontación de ambos y, a través de ellos, construyen sus propias percepciones, de ahí la importancia de reflejar en sí mismo y generar en los estudiantes procesos de motivación, en este caso específicamente hacia la matemática.

\section{Construcción de conocimientos mediante procesos adaptativos.}

El aprendizaje es un proceso personal, pues nadie puede aprender si no es por sus propios medios. Lo anterior no implica que los sujetos de la sociedad no sean elementos que promuevan, faciliten y se involucren en el proceso de aprender. Sin embargo, todos son medios para llegar al objetivo de generar el aprendizaje.

Ante esta perspectiva los profesores, padres y compañeros, entre otros, son facilitadores y mediadores de aprendizaje, pero el único que es capaz de realizar los procesos de asimilación, adaptación y equilibrio es el o la estudiante en sí. 


\section{Revista Cientifica Digital ISSN:2215-2288}

[Número publicado el 30 de Junio del 2012]

URL:http :// revista digita l.eae.fcs.ucr.ac.cr/

\section{Acercamiento de la matemática con la realidad.}

Tomando en cuenta otros aspectos mencionados con anterioridad la cercanía del proceso educativo de la matemática con la realidad es fundamental y, por lo tanto, la resolución de problemas debe estar sujeta, en la mayoría de los casos, a la cotidianidad. No se trata de resolver operaciones, de memorizar contenido, de resolver ecuaciones; se trata de que todo el conocimiento y las experiencias se desarrollen en el margen de lo cotidiano, lo cercano al estudiante, lo útil y aplicable, a los conocimientos que ya conoce y que pueden, junto con otras estrategias, generar conocimientos nuevos.

Debe representar un reto a las capacidades de quien intenta resolverlo, y ser interesante en sí mismo, para poner en manifiesto técnicas, habilidades, estrategias y actitudes personales. Debe tener en cuenta el proceso y no solo el resultado, el método por el cual se logran las cosas, no solo el producto final; el aprendizaje construido, no solo la nota alcanzada; las estrategias utilizadas, no solo los datos obtenidos; las habilidades, destrezas y actitudes desarrolladas, no solo la memorización y el contenido; el trabajo individual y en equipo de los y las estudiantes, no solo los aportes del profesor.

\section{Utilización de un lenguaje matemático.}

En un mundo como el nuestro donde la comunicación y las estrategias para comunicarse cobran tanta importancia y donde el contenido fluye, cambia y se accesa con tanta facilidad, es indispensable aprovechar, refinar y utilizar las estrategias de comunicación más variadas y posibles, en todas las áreas del conocimiento y específicamente en el de la matemática.

El uso diario de un lenguaje matemático acerca al estudiante al medio real, lo sensibiliza, le da herramientas para su empleo en las actividades cotidianas, le permite desarrollar estructuras intelectuales, le brinda conocimiento y le facilita los procesos de descodificación de la información.

El lenguaje matemático debe ser empleado por las docentes de manera constante y debe ser promovido por los estudiantes de igual manera. El uso de un lenguaje matemático empodera al estudiante dentro de los procesos educativos tanto dentro y fuera del aula, le da mayor seguridad y armoniza aspectos teórico-prácticos. Es una herramienta que aporta muchos 


\section{Gị̂Gestión \\ Escuela de Administración Educativa}

[Número publicado el 30 de J unio del 2012]
Revista Cientifica Digital ISSN:2215-2288

URL:http :// revista digita l.eae.fcs.ucr.ac.cr/

beneficios y que rompe barreras de tiempo, espacio y edad. Asimismo, une y permite la interacción más fluida del educando con el profesor, con los especialistas y con el medio.

\section{Análisis, visitas y uso de técnicas de programas y proyectos exitosos.}

La perseverancia, una visión clara de lo que se desea lograr, una misión detalladamente definida, la experiencia y los procesos constantes de evaluación han hecho que muchos proyectos educativos en el área de la matemática sean exitosos.

Analizar esos programas o proyectos, realizar visitas a instituciones que los aplican, buscar información actualizada respecto de ellos, tratar de implementar las estrategias que mejor se adapten a la institución o al grupo de estudiantes con los que se trabaja, son algunas de las actividades que se pueden realizar para tener un acercamiento a proyectos que han generado cambios significativos. No se trata de copiar modelos, sino de analizarlos, adaptarlos y mejorarlos. También, se trata de crear los propios modelos adaptados a las circunstancias particulares en las que se desarrollan los procesos educativos.

\section{Determinar el papel de los y las estudiantes y de los y las docentes.}

Existen muchos elementos que influyen en el proceso educativo, uno de ellos es el docente, especialista cercano al estudiante durante todo el proceso lectivo.

Por lo general, el docente de preescolar comparte entre tres y media y cuatro horas con las niñas y los niños, el docente de primaria comparte entre cuatro y seis horas, a excepción de aquellos quienes se especializan en impartir una o dos materias y que permanecen lapsos menores con sus estudiantes y quienes, además, tienen a su cargo dos o más grupos de niños.

Asimismo, en los ciclos de secundaria los profesores laboran alrededor de cuarenta lecciones de acuerdo con su área de especialización. Por otra parte, el docente universitario

imparte entre dos o cinco horas semanales o quincenales (de acuerdo con la universidad) en un curso especializado para futuros docentes de educación preescolar (entre otros niveles educativos).

El papel del docente es fundamental, tanto desde una visión amplia o restringida del currículo, siempre tendrá un papel, una función y una misión dentro del proceso educativo. Hay que rescatar que dentro de este proceso hay tres formas básicas en las cuales se puede 


\section{Revista Cientifica Digital ISSN:2215-2288}

[Número publicado el 30 de Junio del 2012]

desenvolver un educador:

- Ente autoritario: que delimita los objetivos, actividades y estrategias de evaluación de manera lineal, teniendo únicamente como bases sus propios intereses, necesidades y metas. Al desempeñar este papel, por lo general el sistema que prevalece es propio (el del docente) y el sistema de normas es impuesto.

- Ente sin rumbo fijo "laisser faire, laisser passer": que deja hacer y deja pasar. No existen objetivos, metas y estrategias de evaluación claramente delimitadas y predefinidas; no se sabe a dónde se desea llegar, hasta permite que cualquier persona establezca metas. Muchas veces no es respetado por sus alumnos y no tiene un papel de autoridad.

- Ente democrático: que promueve la participación, escucha la opinión de los demás, integra a los miembros del aula y muchas veces hasta los de la comunidad institucional y local. Toma en cuenta a los estudiantes en las etapas de planificación, desarrollo y evaluación.

Este papel depende mucho de la personalidad del docente, del modelo educativo en el que este fue formado, del medio sociocultural en el que se desarrolla el proceso de enseñanza (etnia, cantón, país), así como de los procesos de formación docente en el que estuvo participando.

El asumir la necesidad de cambios en la forma en que se caracteriza la enseñanza de las matemáticas conlleva una nueva definición del trabajo del profesor y su papel en el aula, por lo tanto, debe ser motivador, dinámico e investigador. Está interesado en producir cambios, es visionario, colaborador en el proceso educativo, reconoce que no hay una sola vía universal de aprender, ni de enseñar; aporta ideas, recursos, materiales, hasta mediador; parte de los conocimientos previos del estudiante, promueve un verdadero trabajo en equipo, promueve la iniciativa y la creatividad, labora con responsabilidad, rompe esquemas, trata de lograr un cambio de paradigma, produce un cambio a través de nuevas técnicas en educación, promueve la participación de la familia, la comunidad y la sociedad, aprovecha recursos humanos y materiales, promueve y desarrolla experiencias significativas, parte de los intereses y necesidades de las estudiantes, es abierto a la crítica, así como promueve habilidades comunicativas. 
Los postulados que orientan el papel del o la estudiante en el curso de matemática para la formación de docentes de educación preescolar, en el nivel universitario, se definen de acuerdo con el tipo de método que orienta el curso. Sin embargo, se propone que sea bajo la óptica de una metodología participativa y, por lo tanto, el o la estudiante de educación preescolar que se matricula y participa en este curso debe ser sujeto y objeto de su propio desarrollo, participante de un proceso integrado e integrador, colaborador en el proceso educativo, aporta de ideas, recursos, materiales, respeta la normas y participa equitativamente en trabajo en equipo, participa en el proceso con iniciativa y creatividad, relaciona conceptos con experiencias diarias. Aprovecha recursos humanos y materiales, promueve, participa y desarrolla experiencias significativas, desarrolla estrategias para la búsqueda y análisis de información por los diversos medios existentes en la actualidad. Crea y elabora materiales, muestra motivación intrínseca y organiza el tiempo para obtener mejores resultados.

El o la estudiante es un ser integral y, como tal, los procesos de enseñanza-aprendizaje de la matemática deben tomar en cuenta su condición y desarrollo físico, social, intelectual y emocional, sus habilidades y limitaciones, sus intereses, sus conocimientos previos, su capacidad para comunicarse utilizando diferentes tipos de expresiones, así como todas aquellas posibilidades que ofrece el medio. Al respecto, la administración de la educación en los actuales escenarios emergentes debe articular las necesidades del educando y las oportunidades nacionales, de manera que se logre contribuir con la transformación de la educación según la emergente sociedad; criterios expuestos por Garbanzo y Orozco (2010), quienes reconocen este papel de la administración de la educación:

El liderazgo en una gestión moderna de la educación siempre reconoce las exigencias que el entorno le demanda, articula el proyecto educativo con las necesidades y oportunidades nacionales, transformando así la educación en un instrumento pertinente de cohesión social y movilidad. Esta gestión le presta particular atención a las variables que interactúan en la consecución del proceso de enseñanza-aprendizaje en forma integrada, con una visión principalmente macro del resultado educativo. (p. 27)

Definitivamente no hay propuestas educativas en aras a contribuir en los procesos de enseñanza-aprendizaje que no requieran del liderazgo acertado de eficientes gestores, que asuman el proyecto educativo con responsabilidad social y actitud propositiva, en busca de los 
cambios y transformaciones sociales que requiere la sociedad, con el propósito de encontrar una mayor equidad e inclusión social.

\section{Conclusiones}

Los procesos de gestión de la educación en el área de la matemática en particular y de la educación en general, deben abocarse al ámbito más amplio posible, que retome todos los actores que intervienen en cada proceso. Las estrategias y los cambios instructivos, formativos y curriculares son fundamentales para lograr el mejoramiento de los procesos educativos; sin embargo, estos van muy relacionados con la organización institucional y por lo tanto dependen de ella. Es precisamente este un campo de acción desde donde la administración de la educación asume relevancia en su rol directivo liderando los procesos organizacionales en los centros educativos, de manera que se implemente con alto nivel de responsabilidad y eficiencia las propuestas educativas necesarias para el proceso enseñanza-aprendizaje, en este caso específico se hace referencia a la enseñanza de la matemática en la educación inicial.

En el caso de la educación matemática a nivel inicial se requiere un cambio en la cultura matemática escolar, en el papel de los conocimientos y creencias previas, en los programas de formación de docentes los cuales deben capacitar a los futuros profesores para que sean capaces en su práctica de caracterizar esa nueva cultura matemática escolar, diferente de la que proceden, un conocimiento de y sobre la actividad matemática, de la historia y epistemología de la disciplina, un conocimiento del currículo matemático escolar, un conocimiento de los estudiantes y de los procesos de aprendizaje, además debe articularse a través de la práctica, por medio de métodos activos que acerquen al estudiante con la realidad, propiciar un ambiente físico y emocional agradable y acogedor, promover procesos motivacionales, y la utilización de un lenguaje matemático, así como analizar, visitar y emplear técnicas de programas y proyectos exitosos. Al respecto, y tomando en cuenta lo señalado en este estudio, en la necesidad de un cambio de cultura, la administración de la educación está llamada a propiciar este tipo de cambios tomando en cuenta las características de cada centro educativo.

Entre las recomendaciones que arrojan la investigación cabe resaltar para los docentes en formación y docentes en ejercicio el promover la participación de los docentes en procesos de formación continua en el área de la matemática, evaluar constantemente los procesos de 


\section{Revista Cientifica Digital ISSN:2215-2288}

[Número publicado el 30 de Junio del 2012]

URL:http:// revista digita l.eae.fcs.ucr.ac.cr/

formación y autoformación en los que participa, de manera tal que estos le sirvan para mejorar los procesos de enseñanza-aprendizaje de la matemática en su aula, desarrollar el hábito y la necesidad de tener acceso a herramientas de información en aspectos relacionados con la matemática (acceso a revistas impresas y electrónicas, Internet, boletines, videos, entre otros medios), promover la revisión y comprensión de la práctica docente cotidiana como un eje de indagación, investigación y autoformación profesional. De esta forma, se evidencia la importancia de los procesos de desarrollo profesional y evaluación de estos mediante eficientes procesos de gestión según los proyectos institucionales.

Se recomienda a universidades, institutos de investigación y medios de comunicación divulgar los resultados obtenidos en investigaciones y trabajos finales de graduación por medio de charlas, talleres, videoconferencia y medios de comunicación masiva, así como promover reuniones, mesas de discusión sobre los principales temas de formación en el área de la matemática; difundir las principales conclusiones, temas, o logros de investigaciones y trabajos finales de graduación en instituciones, empresas, personas físicas o jurídicas interesadas en el mejoramiento del proceso de enseñanza-aprendizaje de la matemática en la educación inicial.

Promover la investigación relacionada con la disciplina, la metodología y los procesos de aprendizaje de la matemática son ejes de los programas de formación docente inicial a considerar y promover una formación matemática teórico-práctica, en contacto con la realidad.

Cabe, también, destacar la responsabilidad que tienen las o los docentes en general en el proceso de autoformación y de formación continua, ya que no todos los recursos de información y capacitación van a llegar a sus manos, sino que él o ella tiene la responsabilidad de buscarlos, obtenerlos, adquirirlos, además de analizarlos y utilizarlos. Lo anterior, presenta una importante articulación entre la responsabilidad docente y las competencias de la administración de la educación en operacionalizar estos procesos de formación continua en sana concordancia con los fines y objetivos de la educación costarricense.

\section{Referencias bibliográficas}

Álvarez, Fernández y Ruiz. (2011). El saber de las docentes en el área de español en las escuelas Río Cañas de Carrillo y Montenegro de Bagaces. Trabajo Final de Graduación para optar por el título de Licenciatura en Educación Primaria. Universidad de Costa Rica.

Barreto, C.; Gutiérrez, L.; Pinilla, B. y Parra, C. (2006). "Límites de constructivismo pedagógico". Educación y educadores, 9(1), pp.11-31. Recuperado de: 
http://redalyc.uaemex.mx/pdf/834/83490103.pdf

Camacho, M. M. (2005). Mejoramiento del proceso de la enseñanza- aprendizaje de la Matemática en la Educación Preescolar, vista desde la perspectiva de la formación docente. Investigación del Instituto de Investigaciones Educativas (INIE), Universidad de Costa Rica. San José, Costa Rica.

Consejo Interamericano para el Desarrollo Integral [CIDI]. (2001). Informe final de la II Reunión de Ministros de Educación. Recuperado de: www.oas.org/udse/3ministerial/contenidos/.../INFORMEFINAL.doc

Darceles, M. (2007). "Gestión del conocimiento y creatividad". Revista Escuela de Administración de Negocios, 61, setiembre-diciembre 2007, pp. 97-102. Recuperado de: http://redalyc.uaemex.mx/src/inicio/ArtPdfRed.jsp?iCve=20611495010

Esquivel, J.; Delgado, V. y Peralta, T. (1983). Diagnóstico evaluativo de la enseñanza de la matemática en la Educación General Básica y Educación Diversificada. Informe final del proyecto de investigación. Universidad de Costa Rica. San José, Costa Rica.

Gamboa, Z. (1994). Educación científica y matemática para el niño preescolar. San José: Editorial EUNED.

Garbanzo, G. y Orozco, V. H. (2010). "Liderazgo para una gestión moderna de procesos educativos". Revista Educación, 34(1), pp. 15-29. Recuperado de: http://www.revistaeducacion.ucr.ac.cr/index.php/educacion/article/viewFile/160/249

Gil P., D. y Guzmán O., M. (1991). Enseñanza de las ciencias y las matemáticas: tendencias e innovaciones. Organización de los Estados Iberoamericanos [OEI]. Recuperado de: http://www.oei.org.co/oeivirt/ciencias.htm

Gonzaga, W. (2005). Las estrategias didácticas en la formación de docentes de educación primaria, Revista Actualidades Investigativas en Educación, 5(1), enero-julio. Recuperado de: http://redalyc.uaemex.mx/pdf/447/44750103.pdf

Gutiérrez C., L. A. (2002). Didáctica de la Matemática para la formación Docente. Coordinación Educativa y cultural Centroamericana. Cartago, Costa Rica: Impresora Obando.

Hernández, A.; Montenegro, M.; Francis, S. y Gonzaga, W. (2002). Estrategias didácticas empleadas en la enseñanza del área pedagógica, en los planes de formación inicial de docentes para la Educación Primaria, en las universidades estatales costarricenses. San José: CECC.

Hurlock, E. B. (1999). Psicología de la adolescencia. México: Paidós.

Leibowicz, J. (2000). Ante el imperativo del aprendizaje permanente, estrategias de formación continua. Montevideo: Cinterfor.

Martín, F. (1999). La didáctica ante el tercer milenio. Madrid, España: Editorial Síntesis.

Organización de Estados Iberoamericanos [OEI]. (1991). El currículo matemático.

Onnetto, H. (2004). "Constructivismo en Psicología". Revista Semestral Pharos: Arte, Ciencia y Tecnología, 11(1), mayo-junio, pp. 37-49. Recuperado de: http://redalyc.uaemex.mx/pdf/208/20811105.pdf

Orozco, D. (2000). Propuesta de Costa Rica perfil de formador de docentes de educación primaria o básica. Proyecto Apoyo al mejoramiento de la formación inicial de docentes de la Educación primaria o básica. San José, Costa Rica: CECC.

Santiago, J. (2006). "Teorías pedagógicas y enseñanza de la geografía en Venezuela". Geoenseñanza, 11(2), julio-diciembre 2006, pp. 145-162. Recuperado de: http://redalyc.uaemex.mx/pdf/360/36012425002.pdf

Tobón, S. y Núñez, A. (2006). "La gestión del conocimiento desde el pensamiento complejo: un compromiso ético con el desarrollo humano". Revista Escuela de Administración de Negocios, 58, setiembre-diciembre, pp. 27-39. Recuperado de: 
http://redalyc.uaemex.mx/pdf/206/20605803.pdf

UNESCO. (2000). Foro mundial de educación para todos. Recuperado de: http://unesdoc.unesco.org/images/0012/001211/121147s.pdf

Venegas, M. (2001). Perfiles y planes de estudio del docente de la educación primaria o básica de los países de Centroamérica: estudio comparativo. San José, Costa Rica: CECC.

Venegas, M. (1996). Formación inicial de docentes de educación primaria o básica. Estudio nacional de Costa Rica. San José, Costa Rica: CECC.

Venegas J., P. (Noviembre 2005). Perspectiva social, liderazgo y retos educativos de los administradores de la educación del sistema educativo costarricense: una experiencia mediante uso de la videoconferencia. II Congreso Internacional de Administradores de la Educación. Escuela de Administración Educativa, Facultad de Educación, Universidad de

Costa Rica.

Recuperado

de: www.eae.ucr.ac.cr/.../Ponencia.\%20Dr.\%20Pedro\%20Venegas.doc

Zambrano, M. (2002). L'Art de les Mediacions (Textos pedagógicos). Barcelona: Universidad de Barcelona. 